\title{
CARACTERIZAÇÃO E AVALIAÇÃO DA REDUÇÃO CARBOTÉRMICA VIA TERMOGRAVIMETRIA DE PÓ DE ACIARIA ELÉTRICA*
}

\author{
Felipe Buboltz Ferreira \\ Bruno Deves Flores ${ }^{2}$ \\ Pedro Jorge Walburga Keglevich de Buzin ${ }^{2}$ \\ Nestor Cezar Heck ${ }^{3}$ \\ Eduardo Osório ${ }^{3}$ \\ Antônio Cezar Faria Vilela ${ }^{4}$
}

\section{Resumo}

O Pó de Aciaria Elétrica (PAE) é um resíduo sólido originado da fabricação de aços em Fornos Elétricos a Arco (FEA). Este resíduo contém metais nocivos ao meio ambiente e é constituído, em sua maior parte, pelos elementos ferro, zinco e oxigênio. Devido aos custos crescentes para disposição e por tratar-se de um resíduo perigoso, a indústria procura tanto opções de reciclagem quanto a possibilidade de retorno ao processo produtivo do aço. Uma das alternativas é a reintrodução na aciaria elétrica através de aglomerados autorredutores como parte da carga do FEA. Nesse trabalho, são confeccionadas misturas autorredutoras contendo PAE e coque de petróleo, apresentando as correspondentes caracterizações químicas e físicas. É feita também uma avaliação do comportamento destas misturas em ensaios termogravimétricos, mostrando a possibilidade do emprego desta técnica na aferição prática do teor ótimo de coque.

Palavras-chave: Redução carbotérmica; PAE; Autorredução; Termobalança.

\section{CHARACTERIZATION AND EVALUATION OF CARBOTHERMIC REDUCTION VIA EAF DUST THERMOGRAVIMETRIC TEST}

\section{Abstract}

The Electric Arc Furnace Dust (EAFD) is a solid waste originated from electric steelmaking furnaces. It has harmful metals to the environment and its made, in majority, by elements iron, zinc and oxygen. Due to increasing costs for disposal and because is considered a hazardous waste, industry look for ways of recycling as well as possibly return the dust to the steelmaking process. One of the alternatives is by reintroducing the waste in electric melt shop using self-reducing agglomerates as part of the furnace burden. In this study, self-reducing mixtures are prepared with EAF Dust and petroleum coke, presenting chemical and physical characterizations. An evaluation about these mixtures' behavior is carried out in thermobalance, regarding the possibility of use by the technique in measuring accurate coke content. Keywords: Carbothermic reduction; EAF Dust; Self-reduction, Thermobalance.

Eng. Materiais, PPGE3M, Universidade Federal do Rio Grande do Sul (UFRGS), Porto Alegre, RS, Brasil.

Me. Eng. Metalúrgico, PPGE3M, UFRGS, Porto Alegre, RS, Brasil.

Prof. Dr. Eng. Metalúrgico, PPGE3M, UFRGS, Porto Alegre, RS, Brasil.

Prof. Dr.-Ing. Metalúrgico, PPGE3M, UFRGS, Porto Alegre, RS, Brasil. 


\section{INTRODUÇÃO}

De acordo com o relatório de sustentabilidade, divulgado pelo Instituto Aço Brasil em 2013, $14 \%$ de coprodutos e resíduos gerados pela indústria siderúrgica, são pós e finos. Dentre estes resíduos se encontra a Poeira de Aciaria Elétrica (PAE) [1]. A produção de aço através de Fornos Elétricos a Arco (FEA) aumentou cerca de $13 \%$ no Brasil e $22 \%$ no mundo, de 2004 a 2013 [2]. Portanto a quantidade de geração de PAE também aumentou. No estado atual da técnica, de acordo com alguns autores, estima-se a geração de 15 a $25 \mathrm{~kg}$ de poeira por tonelada de aço produzido [3-5].

A formação das poeiras de aciaria está associada as seguintes etapas do processo: carregamento do forno, volatilização de metais, como o zinco, vaporização de ferro abaixo do arco, arraste de partículas sólidas e, principalmente, pelo colapso das bolhas de $\mathrm{CO}$ formadas pela descarburação do banho metálico [5]. O PAE é principalmente composto por $\mathrm{Fe}$ e $\mathrm{Zn}$, na forma de diferentes óxidos metálicos, tais como $\mathrm{ZnO}, \mathrm{ZnFe}_{2} \mathrm{O}_{4}, \mathrm{Fe}_{3} \mathrm{O}_{4}$ [6]. Para pós com baixa concentração de zinco, a maior parte deste metal está presente na forma de espinélios ou ferritas. Termodinamicamente, a fase Zincita, aparece no PAE, quando $\mathrm{Zn} /(\mathrm{Fe}+\mathrm{Zn})$ é maior que 0,37 em massa [7]. Conforme aumenta o teor de $\mathrm{Zn}$, aumenta o teor de $\mathrm{ZnO}$ [3]. A composição química do PAE depende fundamentalmente das matérias-primas empregadas, do tipo de aço fabricado e elementos de liga utilizados [3]. Pode variar, sofrendo alterações não somente de um dia para o outro, mas de corrida para corrida em uma mesma usina [5].

Sob o ponto de vista ambiental, de acordo com a NBR 10004, o PAE é classificado como resíduo classe 1, perigoso [8]. Em razão disto, procurando-se evitar a destinação em aterros, busca-se alternativas através da reciclagem ou retorno ao processo produtivo do aço. Tanto a reciclagem como o retorno ao processo baseiam-se tanto na recuperação do conteúdo metálico existente nas poeiras (Fe e $\mathrm{Zn}$, principalmente) quanto na busca da sustentabilidade e redução de custos de destinação ambientalmente adequados para esse resíduo. Dentre os processos pirometalúrgicos de reciclagem, existe atualmente em operação no Brasil a tecnologia Waelz. Este processo baseia-se na redução seletiva das poeiras em um forno tubular, formando-se óxido de zinco impuro, do qual é posteriormente recuperado zinco metálico. Entretanto há somente uma planta em operação, localizada no estado de MG, o que torna essa alternativa dispendiosa para usinas mais distantes. Tendo em vista que em usinas semi-integradas não há reatores de redução, nestes locais existe a dificuldade de reciclar o PAE. Alguns autores $[9,10]$ têm sugerido que nesses casos a recuperação de resíduos ferrosos pode ser realizada através da utilização de aglomerados autorredutores como parte da carga de fornos elétricos a arco.

A tecnologia de autorredução consiste na mistura íntima entre o material carbonáceo e o óxido do qual pretende se extrair o elemento de valor econômico. Os óxidos de ferro e o redutor encontram-se próximos um do outro, num mesmo agregado compacto, com grande área superficial disponível para as reações necessárias a redução. O contato íntimo entre as partículas, deve-se ao fato de que a mistura autorredutora é aglomerada com os constituintes em granulometria relativamente fina [9;10], geralmente utilizando-se de um ligante auxiliar. Uma possibilidade para a formação desses agregados é através da pelotização. Neste processo, um dos requisitos para maior eficiência é que o material a ser pelotizado deva estar em granulometria abaixo de $0,15 \mathrm{~mm}$, sendo que $80 \%$ desses deve ser menor que 
0,045 mm. A diminuição da granulometria do redutor, também, é responsável pelo aumento da taxa de redução do óxido de ferro em uma dada temperatura. Isto se deve ao aumento da superfície específica, a qual potencializa a reação de Boudouard, pelo fato de que as partículas de carbono possuem maior superfície para reagir com o $\mathrm{CO}_{2}$ [11]. No caso específico dos pós de aciaria, a utilização de aglomerados autorredutores pode tornar possível tanto a reciclagem do conteúdo metálico de ferro das poeiras quanto a geração de uma nova poeira com teor enriquecido de zinco, já que nas condições do processo esse metal tende a acumular-se novamente na poeira. Pós com teores maiores de zinco podem ser mais atrativos para posterior comercialização como coproduto e/ou posterior reciclagem.

Tendo em vista o interesse crescente da reciclagem do PAE em aciarias semiintegradas, o objetivo desse trabalho foi a caracterização da poeira e do coque de petróleo, bem como a avaliação do comportamento de autorredução da mistura desses constituintes por termogravimetria. Esse estudo busca dar suporte a reciclagem do PAE através do uso de aglomerados autorredutores em forno elétrico a arco.

\section{MATERIAIS E MÉTODOS}

Para atingir o objetivo do estudo, amostras de pó de aciaria elétrica e coque de petróleo (PET) foram coletadas e caracterizadas. Na sequência são apresentadas as técnicas utilizadas, de caracterização química e física, além da descrição dos ensaios autorredutores em termobalança de misturas de PAE/PET.

\subsection{Caracterização Química e Física do PAE}

O pó de aciaria elétrica utilizado no estudo foi amostrado em uma usina semiintegrada brasileira, a qual produz aços longos ao carbono. A caracterização química do PAE foi feita através de espectrometria de emissão atômica por plasma acoplado indutivamente (ICP), espectroscopia Mössbauer e difração de raios $X$ (DRX). O teor de carbono do PAE foi determinado via combustão seca em analisador elementar CNHS.

Realizou-se também a caracterização física do PAE através das análises de distribuição de tamanho de partícula por difração de laser, de área superficial pelo método BET (Brunauer, Emmett e Teller) e microscopia eletrônica de varredura.

\subsection{Caracterização Química e Física do Coque de Petróleo}

A amostra de PET utilizada nesse estudo foi obtida junto à mesma usina fornecedora do PAE. Devido à granulometria de recebimento do coque de petróleo ser considerada grosseira $(50 \%>0,5 \mathrm{~mm})$ para os fins desse estudo (posterior produção de pelotas), realizou-se a adequação granulométrica em moinho de bolas. Após a moagem do material a caracterização do PET foi realizada através das análises imediata e distribuição de tamanho de partícula por difração de laser.

\subsection{Ensaios Termogravimétricos de Misturas PAE/PET}

Foram realizados ensaios em termobalança com misturas contendo PAE e PET, conforme mostra a Tabela 1. Os ensaios foram realizados em uma termobalança 
Netzsch, modelo 409 PC Luxx. Misturas, com massa de 250 mg, foram introduzidas em cadinho de alumina tipo copo $(37 \times 54 \mathrm{~mm})$, onde foram ligeiramente compactadas de forma manual. As amostras foram aquecidas a uma taxa de $30^{\circ} \mathrm{C} / \mathrm{min}$ até a temperatura de $600^{\circ} \mathrm{C}$, e mantidas nesta temperatura por $20 \mathrm{~min}$. Essa primeira etapa isotérmica foi realizada com o intuito de eliminar das amostras a umidade e grande parte da matéria volátil do agente redutor, evitando assim perdas de massa devido à redução dos óxidos do PAE. Após essa etapa de desvolatilização, as amostras foram aquecidas a uma taxa de $30^{\circ} \mathrm{C} / \mathrm{min}$ até $1100^{\circ} \mathrm{C}$ e mantidas nesta temperatura por $20 \mathrm{~min}$. Um fluxo de $100 \mathrm{ml} / \mathrm{min}$ de nitrogênio foi introduzido no forno ao longo de todo o ensaio.

Tabela 1. Composição das amostras utilizadas nos testes de autorredução em termobalança.

\begin{tabular}{cccccccc}
\hline Composição & PAE & 97 & 96 & 95 & 92 & 90 & 85 \\
(\% em massa) & PET & 3 & 4 & 5 & 8 & 10 & 15 \\
\hline
\end{tabular}

\section{RESULTADOS E DISCUSSÃO}

\subsection{Caracterização do Pó de Aciaria Elétrica}

A Tabela 2 apresenta a composição elementar do PAE, obtida via ICP. Os elementos $\mathrm{Zn}(34,23 \%)$ e Fe $(22,80 \%)$ compõem majoritariamente o resíduo. De acordo com a literatura [3], a concentração de $\mathrm{Zn}$ em pós oriundos da fabricação de aços ao carbono pode variar entre 11,12 e 26,9 \%. O teor mais elevado de $\mathrm{Zn}$ no PAE estudado tem como possível causa a maior utilização de sucata galvanizada na fabricação de aço utilizado pela usina onde o pó foi amostrado. O teor de carbono do PAE é de $0,91 \%$. Outros elementos mostrados na Tabela 2 estão dentro das faixas usualmente encontradas na literatura $[3,12]$. Elementos não analisados, dentre eles o oxigênio, completam a caracterização da poeira e contabilizam 31,53 \% do PAE.

Tabela 2. Composição química do PAE.

\begin{tabular}{ccccccccc} 
Composição & $\mathrm{Zn}$ & $\mathrm{Fe}$ & $\mathrm{Mn}$ & $\mathrm{Ca}$ & $\mathrm{Mg}$ & $\mathrm{Pb}$ & $\mathrm{Na}$ & $\mathrm{C}$ \\
elementar do & 34,23 & 22,80 & 2,75 & 2,49 & 1,76 & 1,10 & 1,00 & 0,91 \\
\cline { 2 - 9 } PAE & $\mathrm{K}$ & $\mathrm{Al}$ & $\mathrm{Cu}$ & $\mathrm{Ba}$ & $\mathrm{Cd}$ & $\mathrm{Ni}$ & $\mathrm{Sn}$ & \\
(\% em massa) & 0,97 & 0,20 & 0,15 & 0,05 & 0,03 & 0,01 & 0,01 & \\
\hline
\end{tabular}

A Figura 1 apresenta as espécies cristalinas identificadas no PAE via DRX. Observase que a análise indica a presença de Franklinita $\left(\mathrm{ZnFe}_{2} \mathrm{O}_{4}\right)$, Magnetita $\left(\mathrm{Fe}_{3} \mathrm{O}_{4}\right)$ e Zincita $(\mathrm{ZnO})$. A presença de tais espécies químicas está de acordo com o que poderia se esperar de uma composição típica de poeira de aciaria. No entanto, salienta-se que devido à sobreposição de picos de difração não é possível afirmar, apenas com a análise de DRX, a presença dos minerais identificadas como A e B no difratograma. Estas foram também identificadas em outros estudos de caracterização de pós de aciaria $[13,14]$. 


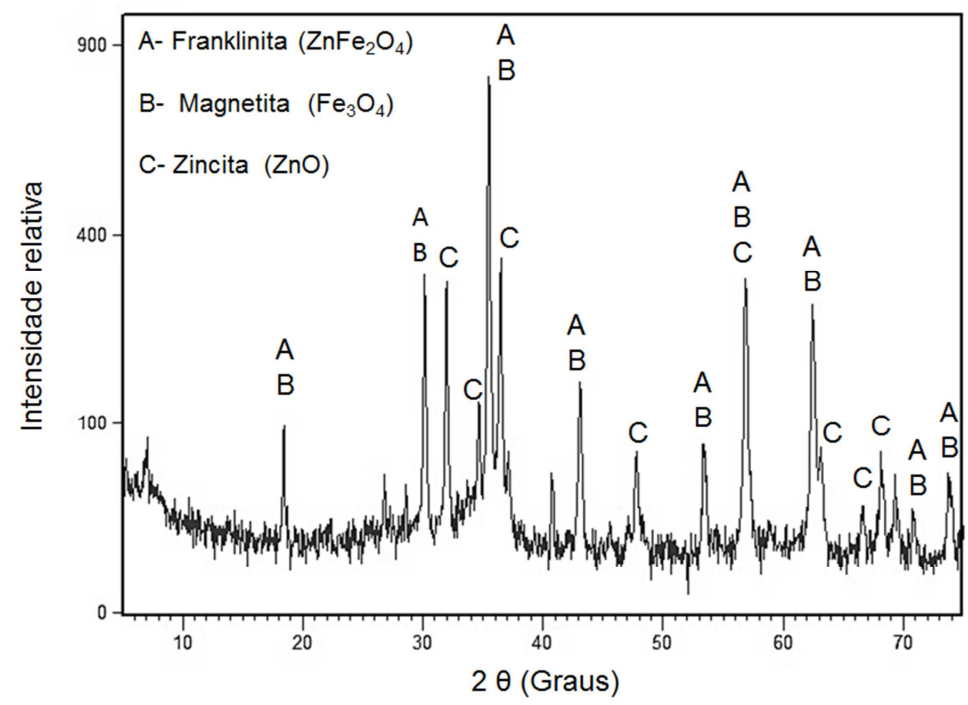

Figura 1. Difratograma de raios $X$ da amostra de PAE.

Para auxiliar na caracterização das fases ferrosas foram realizadas análises via espectroscopia Mössbauer e os resultados estão indicados na Tabela 3. Verifica-se que o ferro presente no PAE (22,8\% - Tabela 2) está distribuido no mineral Franklinita (75\%) e na Magnetita (25\%). Assim, a espectroscopia Mössbauer complementa o resultado da análise via DRX, confirmando a presença tanto de Franklinita como de Magnetita no material.

Tabela 3. Quantificação das fases ferrosas do PAE via espectroscopia Mössbauer.

$\begin{array}{ccc}\text { Distribuição das fases de ferro } & \mathrm{ZnFe}_{2} \mathrm{O}_{4} & \mathrm{Fe}_{3} \mathrm{O}_{4} \\ \text { (\% em massa) } & 75,0 & 25,0\end{array}$

A partir da composição elementar do PAE, indicada na Tabela 2 e da quantificação das fases de ferro, apresentada na Tabela 3, estimou-se os teores das fases óxidas majoritárias presentes no PAE $\left(\mathrm{ZnFe}_{2} \mathrm{O}_{4}, \mathrm{ZnO}\right.$ e $\left.\mathrm{Fe}_{3} \mathrm{O}_{4}\right)$ (Tabela 4).

Tabela 4. Estimativa das fases majoritárias do PAE.

\begin{tabular}{ccccc}
\hline Composição do & $\mathrm{ZnFe}_{2} \mathrm{O}_{4}$ & $\mathrm{ZnO}$ & $\mathrm{Fe}_{3} \mathrm{O}_{4}$ & Outras fases \\
PAE $(\%$ em massa $)$ & 36,9 & 30,2 & 7,9 & 25,1 \\
\hline
\end{tabular}

Essa estimativa foi feita de acordo com o procedimento descrito por Machado et al., conforme apresentado a seguir [15]:

- Estimativa do teor de $\mathrm{ZnFe}_{2} \mathrm{O}_{4}$ :

$\% \mathrm{Fe}_{\mathrm{ZnFe} 2 \mathrm{O} 4}=\frac{\% \mathrm{Fe}_{\text {total }}(\text { vialCP) }}{\mathrm{N} \% \mathrm{Fe}_{\mathrm{ZnFe} 2 \mathrm{O} 4 \text { (viaMössbauer) }}}$

$\% \mathrm{ZnFe}_{2} \mathrm{O}_{4}=\frac{\left.\% \mathrm{Fe}_{\mathrm{ZnFe} 2 \mathrm{O} 4 \mathrm{XM}} \mathrm{Fe}_{\mathrm{ZnFe} 2 \mathrm{O} 4}\right)}{\mathrm{M}_{\mathrm{ZnFe} 2 \mathrm{O} 4}}$

- Estimativa do ZnO:

$\% \mathrm{Zn}_{\mathrm{ZnFe} 2 \mathrm{O} 4=} \frac{\mathrm{ZnFe}_{2} \mathrm{O}_{4} \mathrm{xM} \mathrm{Zn}_{\mathrm{Zn}}}{\mathrm{M}_{\mathrm{ZnFe} 2 \mathrm{O} 4}}$ 
$\% \mathrm{Zn}_{\mathrm{ZnO}=} \% \mathrm{Zn}_{\text {total }}($ via ICP $)-\% \mathrm{Zn}_{\mathrm{ZnFe} 2 \mathrm{O} 4}$

$\% \mathrm{ZnO}_{=} \frac{\% \mathrm{Zn}_{\mathrm{ZnO}} \times \mathrm{M}_{\mathrm{ZnO}}}{\mathrm{M}_{\mathrm{Zn}}}$

Onde:

$\% \mathrm{Fe}_{\mathrm{Zn} 2 \mathrm{O} 4}$ : \%Fe no mineral Franklinita;

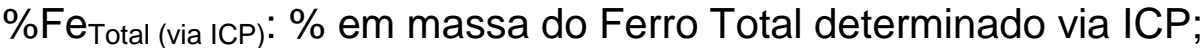

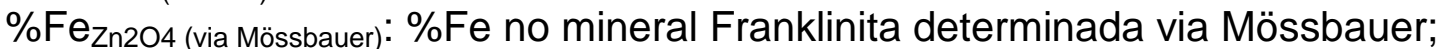

$\% \mathrm{ZnFe}_{2} \mathrm{O}_{4}: \%$ de Franklinita nas amostras;

$\mathrm{M}_{\mathrm{Fe}(\mathrm{Zn} 2 \mathrm{O} 4)}$ : massa molar do Fe no mineral Franklinita;

$\mathrm{M}_{\mathrm{ZnFe} 2 \mathrm{O} 4}$ : massa molar do mineral Franklinita;

$\mathrm{M}_{\mathrm{Zn}}$ : massa molar do $\mathrm{Zn}$;

$\% \mathrm{Zn}_{\mathrm{ZnO}}: \% \mathrm{Zn}$ na fase $\mathrm{ZnO}$;

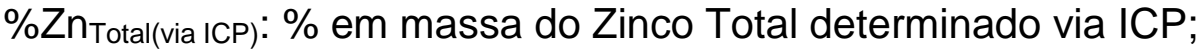

$\mathrm{M}_{\mathrm{ZnO}}$ : massa molar da fase Zincita;

\%ZnO: \% de Zincita nas amostras.

Observa-se que os compostos $\mathrm{ZnFe}_{2} \mathrm{O}_{4}(36,9 \%)$ e $\mathrm{ZnO}(30,2 \%)$ são majoritários, com teores menores de $\mathrm{Fe}_{3} \mathrm{O}_{4}(7,9 \%)$. De acordo com alguns autores [16,17], a quantidade de Zincita é maior em pós de aciaria ricos em zinco, o que explica a alta quantidade de $\mathrm{ZnO}$ no PAE estudado. Compostos formados por elementos que não contenham ferro ou zinco somam $25,1 \%$ do PAE.

A partir dessa estimativa (Tabela 4), calculou-se o teor de oxigênio associado a essas fases de acordo com a equação 1.

$$
\mathrm{O}_{\text {redutivel }}=\% \mathrm{ZnFe}_{2} \mathrm{O}_{4} \cdot \frac{\mathrm{M}_{\mathrm{O}}}{\mathrm{M}_{\mathrm{ZnFe} 204}}+\% \mathrm{ZnO} \cdot \frac{\mathrm{M}_{\mathrm{o}}}{\mathrm{M}_{\mathrm{ZnO}}}+\% \mathrm{Fe}_{3} \mathrm{O}_{4} \cdot \frac{\mathrm{M}_{\mathrm{O}}}{\mathrm{M}_{\text {eе } 304}}
$$

Onde $\mathrm{O}_{\text {redutivel }}$ corresponde ao percentual mássico de oxigênio ligado às espécies químicas $\mathrm{ZnFe}_{2} \mathrm{O}_{4}, \mathrm{ZnO}$ e $\mathrm{Fe}_{3} \mathrm{O}_{4} \mathrm{e} \mathrm{M}$ é a massa molar do oxigênio ou de cada um dos compostos $\left(\mathrm{ZnFe}_{2} \mathrm{O}_{4}, \mathrm{ZnO}\right.$ e $\left.\mathrm{Fe}_{3} \mathrm{O}_{4}\right)$. Assim, estimou-se que o teor de oxigênio redutível do PAE é de 17,9\%. O teor de oxigênio redutível corresponde à quantidade de oxigênio a ser removida por redução carbotérmica para a obtenção de ferro e zinco metálicos. Baseado nesse valor e na estequiometria das reações de redução dos óxidos de ferro e zinco, é possível estabelecer a quantidade mínima necessária de carbono em misturas autorredutoras.

Sabe-se que outros óxidos, geralmente presentes em pós de aciaria, como óxido de chumbo, níquel e cromo, também são passíveis de redução carbotérmica em altas temperaturas [18]. No entanto, o teor de oxigênio redutível estimado pela equação 1, desconsiderou a contribuição desses óxidos, pois eles estão presentes em baixas quantidades no pó estudado.

Além da composição química do PAE, as características físicas do material são importantes no contexto da autorredução. A Figura 2 mostra a distribuição granulométrica do PAE, onde cerca de 80 - $85 \%$ das partículas têm tamanho inferior a $5 \mu \mathrm{m}$. A área superficial BET foi de $3,93 \mathrm{~m}^{2} / \mathrm{g}$, valor dentro da faixa encontrada na literatura [12]. A Figura 3 mostra a morfologia esférica das partículas de PAE. 


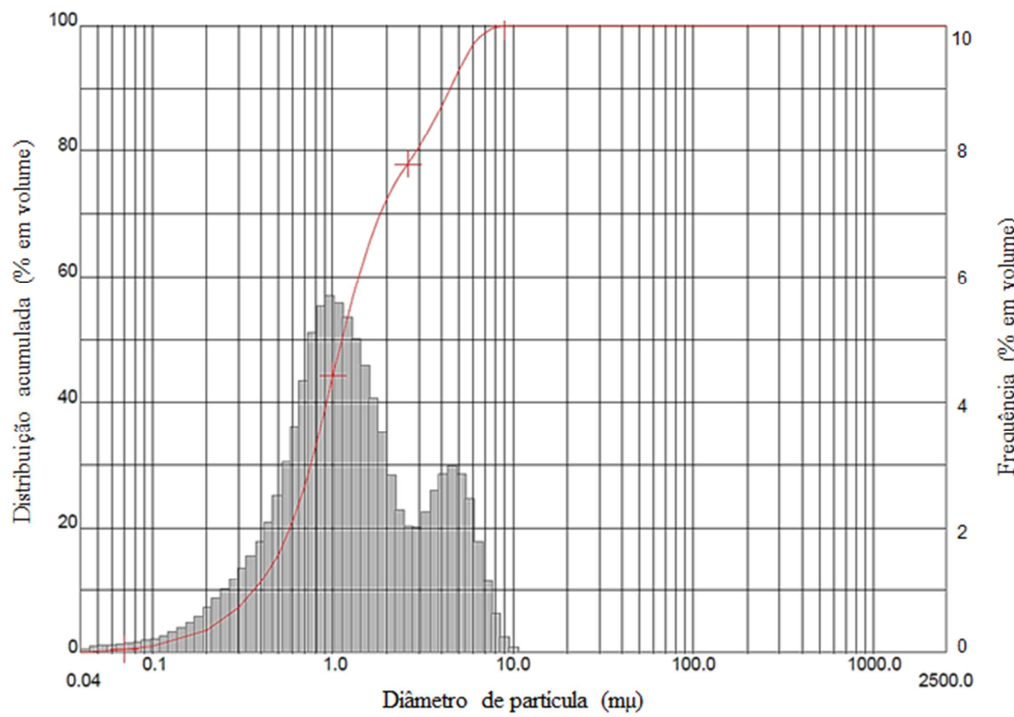

Figura 2. Granulometria do PAE.

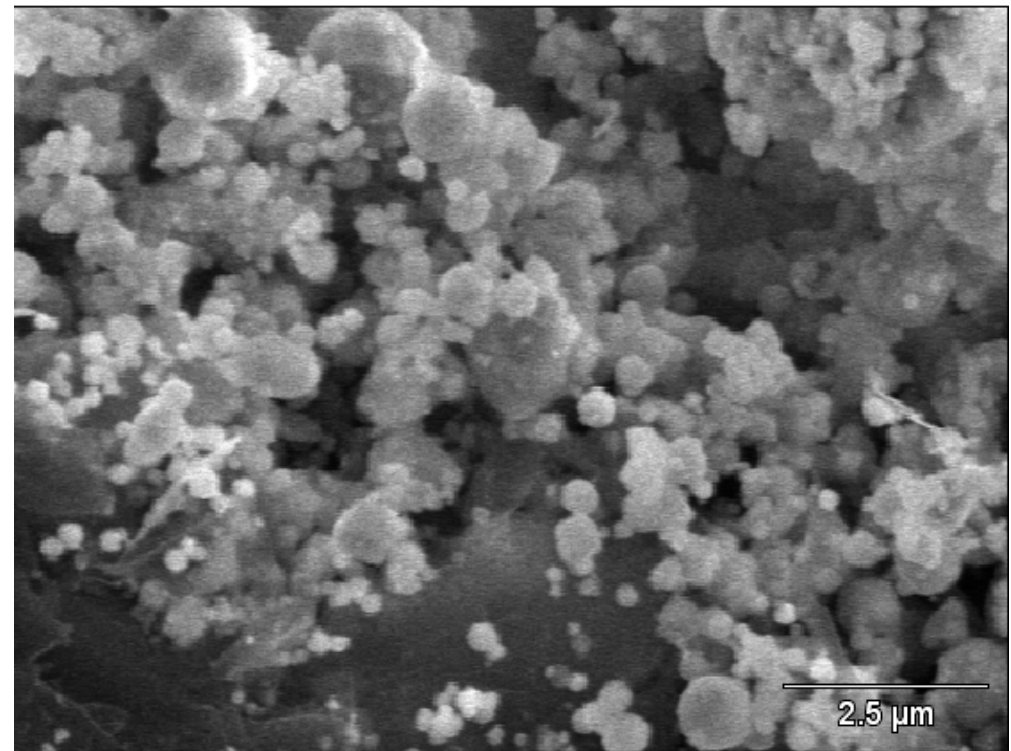

Figura 3. Imagem de Microscopia Eletrônica de Varredura do PAE.

\subsection{Caracterização do Agende Redutor}

A Tabela 5 mostra a análise imediata do coque de petróleo. Este redutor possui um baixo teor de contaminantes inorgânicos $(0,6 \%)$ e um alto conteúdo de carbono fixo. Com vistas ao objetivo global do estudo, a utilização de redutores com baixo teor de cinzas na fabricação de aglomerados autorredutores para a utilização em fornos elétricos a arco, apresenta como vantagem a menor geração de escória. Além disso, o mais alto teor de carbono fixo desse redutor, em relação a outros redutores usualmente empregados em aglomerados autorredutores [19], resulta em misturas com uma relação resíduo óxido/redutor maior.

Tabela 5. Análise imediata do coque de petróleo.

\begin{tabular}{cccc}
\hline Composição & Matéria Volátil & Cinzas & Carbono fixo \\
$(\%$ em massa base seca $)$ & 10,0 & 0,6 & 89,4 \\
\hline
\end{tabular}


A Figura 4 mostra a granulometria do coque de petróleo após processo de moagem. O redutor apresenta aproximadamente $10 \%$ das partículas em tamanho inferior a 10 $\mu \mathrm{m}$ e mais de $50 \%$ do material possui granulometria acima de $100 \mu \mathrm{m}$.

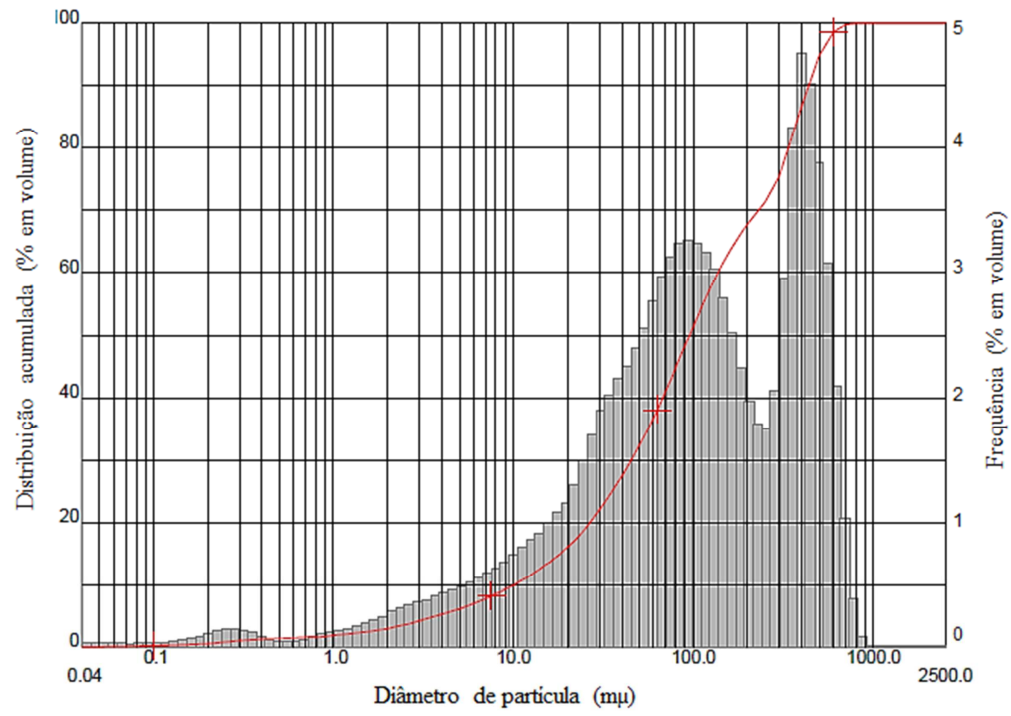

Figura 4. Granulometria do coque de petróleo.

\subsection{Ensaios Termogravimétricos das Misturas de PAE/PET}

A Tabela 6 apresenta as misturas de PAE/PET submetidas a ensaios termogravimétricos com a estimativa dos seus constituintes e as respectivas relações mássicas Carbono/ $O_{\text {redutivel. }}$ Observa-se que ao se aumentar a quantidade de redutor nas misturas as quantidades de oxigênio redutível e zinco devem ser

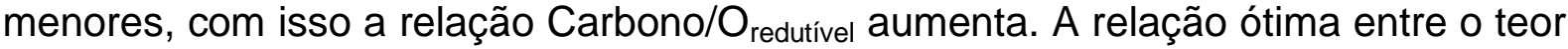
de carbono e o oxigênio a ser reduzido nas misturas é aquela onde a perda de massa é maximizada, com a adição mínima de redutor na mistura.

Tabela 6. Composição das misturas antes dos ensaios termogravimétricos.

\begin{tabular}{|c|c|c|c|c|c|c|c|}
\hline \multicolumn{2}{|c|}{ Misturas } & \multicolumn{3}{|c|}{ Constituintes (\% em massa) } & \multirow[b]{2}{*}{$\begin{array}{c}\text { Carbono/ } \\
\text { Oredutivel }\end{array}$} & \multirow{2}{*}{$\begin{array}{c}\text { Máx. } \\
\text { perda de } \\
\text { massa } \\
\text { estimada }\end{array}$} & \multirow[b]{2}{*}{$\begin{array}{c}\text { Perda total } \\
\text { no ensaio } \\
\text { (\%) }\end{array}$} \\
\hline PAE & PET & $\mathrm{O}_{\text {redutivel }}$ & $\mathrm{Zn}$ & $\begin{array}{c}\text { Carbono } \\
\left(\mathrm{C}_{\mathrm{fixo}}+\mathrm{C} \text { do PAE }\right)\end{array}$ & & & \\
\hline 97 & 3 & 17,4 & 33,2 & 3,6 & 0,20 & 54,2 & 31,7 \\
\hline 96 & 4 & 17,2 & 32,9 & 4,4 & 0,26 & 54,5 & 39,6 \\
\hline 95 & 5 & 17,0 & 32,5 & 5,3 & 0,31 & 54,8 & 40,5 \\
\hline 92 & 8 & 16,5 & 31,5 & 8,0 & 0,48 & 56,0 & 53,5 \\
\hline 90 & 10 & 16,1 & 30,8 & 9,8 & 0,61 & 56,7 & 59,2 \\
\hline 85 & 15 & 15,2 & 29,1 & 14,2 & 0,93 & 58,5 & 59,0 \\
\hline
\end{tabular}

A Figura 5 mostra o resultado dos ensaios termogravimétricos das misturas de PAE/PET. Observa-se que até a primeira etapa isotérmica do teste $\left(600^{\circ} \mathrm{C}\right)$, todas as amostras apresentaram comportamento semelhante. Durante esta primeira etapa as perdas de massa observadas são relativas à liberação da umidade das matériasprimas, bem como a desvolatilização parcial do coque de petróleo. Assim, seria plausível esperar diferenças de perda de massa durante a primeira etapa, uma vez que as misturas apresentam diferentes teores de redutor. No entanto, devido à baixa quantidade de matéria volátil do coque de petróleo e dos pequenos teores de redutor 
nas misturas, não foi possível verificar diferenças significativas entre as amostras. As perdas de massa para todas as amostras foram de aproximadamente $3 \%$.

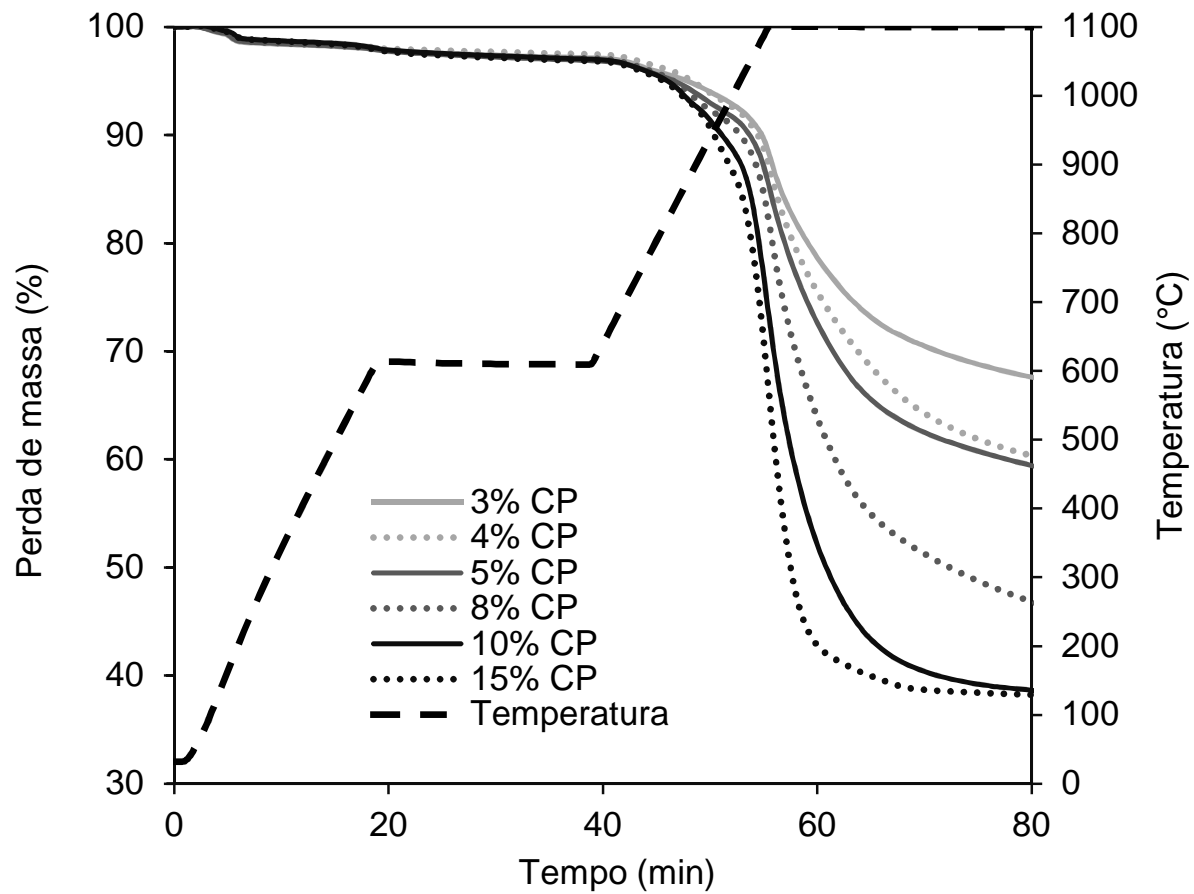

Figura 5. Ensaios termogravimétricos das misturas de PAE e coque de petróleo.

Com o aumento da temperatura observa-se que as amostras apresentaram uma acentuada perda de massa (Figura 5), que se inicia em temperatura próxima a $900^{\circ} \mathrm{C}$ e segue até a temperatura máxima de teste $\left(1100^{\circ} \mathrm{C}\right)$. O comportamento das amostras em temperaturas acima de $900^{\circ} \mathrm{C}$ está vinculado à redução dos óxidos metálicos contendo oxigênio redutível, da gaseificação do carbono fixo presente no agente redutor e principalmente devido à perda de zinco que deixa o sistema na fase gasosa. A perda de massa das amostras devido à vaporização do zinco, é provocada pela redução/vaporização deste metal, uma vez que após a redução, o zinco metálico é imediatamente vaporizado $\left(907^{\circ} \mathrm{C}\right)$.

A redução carbotérmica dos óxidos de ferro e zinco presentes no PAE pode ser representada genericamente pela equação (2). Essa equação estabelece uma relação mássica carbono/oxigênio igual a 0,75 para a total redução dos óxidos de ferro e zinco presentes no PAE. Logo, o comportamento de perda de massa das diferentes amostras no gráfico da Figura 5 pode ser explicado ao se avaliar a relação Carbono/O $\mathrm{O}_{\text {redutivel }}$ de cada uma dessas misturas (Tabela 6). Observa-se que a perda de massa aumenta na medida em que a relação Carbono/ $O_{\text {redutivel }}$ das amostras se aproxima a 0,75. No entanto, verificou-se que as misturas com 10 e 15 $\%$ de PET, tiveram perdas de massa semelhantes. Assim, para as misturas com menores valores de da relação Carbono/Oredutivel, o percentual de carbono foi insuficiente para se atingir a total redução dos óxidos de ferro e zinco. Logo, a perda de massa verificada para as amostras de 3 a $8 \%$ de coque de petróleo nas misturas, é inferior a máxima perda estimada (Tabela 6). Já para as misturas com 10 e $15 \%$ de PET, as perdas verificadas (Figura 5) são próximas à máxima perda estimada. É digno de nota que o resultado de perda de massa para a amostra com $10 \%$ de PET indica a total redução dos óxidos de ferro e zinco presentes no PAE,

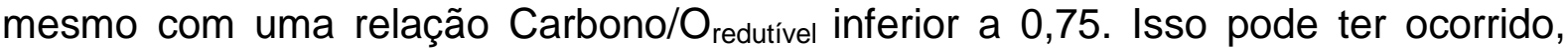
pois a redução dos óxidos metálicos nas temperaturas onde se deu a maior perda 
de massa da mistura, ocorre com formação mista de $\mathrm{CO} / \mathrm{CO}_{2}$, ou seja, com um menor consumo de carbono do que prevê a reação 2 . Verifica-se ainda que a perda de massa das amostras com 10 e $15 \%$ de PE, obtida no final do ensaio, foi ligeiramente superior à máxima estimada. Acredita-se que isso se deva a redução de outros óxidos contidos no PAE e que não foram incluídos na estimativa de máxima perda.

$$
\mathrm{MO}_{(\mathrm{s})}+\mathrm{C}_{(\mathrm{s})}=\mathrm{M}_{(\mathrm{s})}+\mathrm{CO}_{(\mathrm{g})}
$$

Embora as misturas com 10 e $15 \%$ de PET tiveram rendimentos semelhantes, é possível verificar no gráfico da Figura 5 a existência de uma vantagem cinética da amostra com maior quantidade de redutor. A reação (2) mostra a redução dos óxidos ocorrendo através de uma reação do tipo sólido-sólido. No entanto, é consenso entre pesquisadores da área [20,21] que a redução carbotérmica dos óxidos de ferro e zinco, em aglomerados autorredutores, ocorre majoritariamente através de intermediários gasosos, conforme mostra as reações (3) e (4). O gás redutor $\mathrm{CO}$ é formado no interior das misturas a partir da reação de Boudouard, onde 0 carbono presente no agente redutor reage com $0 \mathrm{CO}_{2}$ oriundo das reações de redução, conforme mostra a reação (5). Como as reações de redução dos óxidos de ferro e zinco e a reação de Boudouard ocorrem de forma acoplada, a etapa mais lenta (redução ou gaseificação) controla o processo global de redução do aglomerado. Segundo diversos estudos [21-23] a cinética de redução de aglomerados autorredutores, principalmente em baixas temperaturas, é controlada pela reação de Boudouard. Assim, acredita-se que ao se elevar a proporção de 10 para $15 \%$ de redutor nas misturas ocorre um aumento na cinética de produção de $\mathrm{CO}$, acentuando a velocidade de redução da mistura.

$$
\begin{aligned}
& \mathrm{Fe}_{\mathrm{x}} \mathrm{O}_{\mathrm{y}(\mathrm{s})}+\mathrm{CO}_{(\mathrm{g})}=\mathrm{FexO}_{\mathrm{y}-1(\mathrm{~s})}+\mathrm{CO}_{2(\mathrm{~g})} \\
& \mathrm{ZnO}_{(\mathrm{s})}+\mathrm{CO}_{(\mathrm{g})}=\mathrm{Zn}_{(\mathrm{g})}+\mathrm{CO}_{2(\mathrm{~g})} \\
& \mathrm{C}_{(\mathrm{s})}+\mathrm{CO}_{2(\mathrm{~g})}=2 \mathrm{CO}_{(\mathrm{g})}
\end{aligned}
$$

Sob o ponto de vista de aplicação prática, pode-se dizer que o estudo realizado pode ser utilizado como uma metodologia para determinação do teor de carbono ótimo em formulações da natureza do experimento. Neste sentido, de acordo com a Figura 5, pode-se levar em consideração fatores como a economia na utilização de redutor ou a cinética das reações, para casos em que a perda mássica seja muito próxima, como por exemplo nas misturas de 10 e $15 \%$ de coque de petróleo. Neste caso há opção do uso de teores menores que $15 \%$ de agente redutor. Teores maiores de PET levam ao maior consumo de matéria-prima, porém esse maior percentual tem cinética mais favorável. Isto pode ser vantajoso, por exemplo, em operações em forno elétrico a arco, visando a reintrodução no processo.

\section{CONCLUSÃO}

Após análise dos resultados, destacam-se os seguintes itens:

- Os elementos de interesse econômico, ferro e zinco, estão presentes no PAE em teores de 22,8 e $34,23 \%$, respectivamente.

- Estes elementos estão presentes nas espécies químicas $\mathrm{ZnFe}_{2} \mathrm{O}_{4}(36,9 \%), \mathrm{ZnO}$ $(30,2 \%)$ e $\mathrm{Fe}_{3} \mathrm{O}_{4}(7,9 \%)$. A partir desses valores, estimou-se que o oxigênio redutível do PAE seja de $17,9 \%$. 
- Através dos ensaios de autorredução em termobalança, verificou-se que o teor ótimo de coque de petróleo se situa entre 10 e $15 \%$.

- Considerando-se a maneira de determinação do teor ótimo de coque de petróleo, pode-se salientar o potencial de utilização desta técnica no campo industrial para o ajuste do teor de carbono em lotes de pelotas autorredutoras.

\section{Agradecimentos}

Agradecimentos à CAPES (processo 23078.008775/2014-39), à Denise Souza Bordinhão pelo apoio na realização do trabalho, ao professor Maurício C. Bagatini (UFMG) por sua contribuição e ao Instituto de Física da Universidade Federal do Rio Grande do Sul pela realização do ensaio de espectroscopia Mössbauer.

\section{REFERÊNCIAS}

1 Relatório de Sustentabilidade 2014. Instituto Aço Brasil. Disponível em http://www.acobrasil.org.br/site/portugues/sustentabilidade/downloads/Relatorio\%20de $\% 20$ Sustentabilidade_2014_web.pdf

2 Steel Statistical Yearbook 2014. Disponível em http://www.worldsteel.org/dms/internetDocumentList/statistics-archive/yearbookarchive/Steel-Statistical-Yearbook-2014/document/Steel-Statistical-Yearbook-2014.pdf

3 Machado JGMS, Estudo da Caracterização e Avaliação da Influência do Tempo na Redução Carbotérmica do Pó de Aciaria Elétrica. Dissertação de Mestrado, PPGE3M, Universidade Federal do Rio Grande do Sul, 2004.

4 Alves JO, Espinosa DCR, Tenório JAS., Recycling of Steelmaking Slag Aiming at the Production of Thermo Accoustic Insulation, TMS 2009, 138 th Annual Meeting and Exibithion2009, San Francisco, USA.

5 Guézennec AG, Huber JC, Patisson F, Sessiesc P, Birat JP, Ablitzer D, Dust Formation in Electric Arc Furnace: Birth of the Particles, Powder Technology, 2005.

6 Pickles C A, Thermodynamic Modelling of the Multiphase Pyrometallurgical Processing of electric Arc Furnace. Minerals Engineering. Ontario, Canada, 2009.

7 Buzin PJWK, Heck NC, Vilela ACF, EAFD - A Thermodynamic Analysis and Classification of Dust Types. ABM, 2014.

8 ABNT - Associação Brasileira de Normas Técnicas - Resíduos Sólidos Classificação NBR 10004:2004. Pg. 71. Rio de Janeiro.

9 Buzin PJWK, Desenvolvimento de Briquetes Autorredutores a partir de Carepas de Processamento Siderúrgico para Utilização em Forno Elétrico a Arco. Dissertação de Mestrado, PPGE3M, Universidade Federal do Rio Grande do Sul, 2009.

10 Bagatini MC, Estudo de Reciclagem da Carepa através de Briquetes Autorredutores para uso em Forno Elétrico a Arco. Tese de Doutorado, PPGE3M, Universidade Federal do Rio Grande do Sul, 2001.

11 Nogueira AEA, Estudo da Redução Carbotérmica de Minérios de Ferro na Forma de Pelotas ou Misturas Autorredutoras em Forno Rotativo. Tese de Doutorado, Escola politécnica da USP, 2010.

12 Nyirenda RL, The Processing of Steelmaking Flue Dust: a Review. Minerals Engineering, 1991.

13 Martins FM, Neto JMR, Cunha CJ, Mineral Phases of Weathered and Recent Electric Arc Furnace Dust. Journal of Hazardous Materials, v. 154, 2008.

14 Menad N, Ayala JN, Carcedo FG, Hernandez A, Study of the Presence of Fluorine in the Recycled Fractions During Carbothermal Treatment of EAF Dust. Waste Management, v. 23, 2003.

15 Machado JGMS, Brehm FA, Moraes CAM, Santos CA, Vilela ACF, Characterization Study of Electric Arc Furnace Dust Phases. Materials research, v.9, 2006. 
16 Mantovani MC, Caracterização de Poeiras Geradas em Forno Elétrico a Arco e seu Estudo quando aglomeradas na forma de Pelotas Autorredutoras. Tese de Doutorado. Escola politécnica, Universidade de São Paulo, São Paulo, 1998.

17 Takano C, Cavalamnte FL, Mantovani MC, Mourão MB, Electric Arc furnace Dust Characterization and Recycling by Self-Reducing Pellets, Japan-Brazil Symposium on Dust Processing, São Paulo, 1999.

18 Peng J, Peng B, Yu D, Tang M, Lobel J, Kozinski JB, Volatilization of Zinc and Lead in Direct Recycling of Stainless Steel Making Dust. Trans. Nonferrous Met. Soc. China, v.14, 2004, pp 392-396.

19 Bagatini MC, Zymla V, Osório E, Vilela ACFV, Carbon Gasification in Self-Reducing Mixtures. ISIJ International, v.54, 2014.

20 Sheikhshab BM, Karimi M, Adeli MA Kinetic Study on the Carbothermic Reduction of Zinc Oxide from Electric Arc Furnace Dust, Iranian Journal of Materials Science \& Engineering, V. 10, 2013, pp 18-30.

21 Carvalho RJ; Neto PGQ; D'Abreu JC. Kinetics of reduction of composite pellets containing iron ore and carbon, Canadian Metallurgical Quarterly, Vol. 33, n. 3, p. 217225, 1994.

22 Fruehan RJ. Rate of reduction of iron oxides by carbon. Metallurgical Transactions $B$ Process Metallurgy, v. 8, n. 2, p. 279-286, 1977.

23 Moon J; Sahajwalla V. Investigation into the role of the Boudouard reaction in selfreducing iron oxide and carbon briquettes. Metallurgical and Materials Transactions BProcess Metallurgy and Materials Processing Science, v. 37, n. 2, p. 215-221, Apr 2006. 